\title{
Postweaning exposure to a high-fat diet is associated with alterations to the hepatic histone code in Japanese macaques
}

\author{
Melissa A. Suter ${ }^{1}$, Diana Takahashi², Kevin L. Grove ${ }^{2,3}$ and Kjersti M. Aagaard'
}

BACKGROUND: Expression of circadian gene, Npas2, is altered in fetal life with maternal high-fat (HF) diet exposure by virtue of alterations in the fetal histone code. We postulated that these disruptions would persist postnatally.

METHODS: Pregnant macaques were fed a control (CTR) or HF diet and delivered at term. When offspring were weaned, they were placed on either CTR or HF diet for a period of $5 \mathrm{mo}$ to yield four exposure models (in utero diet/postweaning diet: $\mathrm{CTR} / \mathrm{CTR} n=5 ; \mathrm{CTR} / \mathrm{HF} n=4 ; \mathrm{HF} / \mathrm{CTR} n=4$; and HF/HF $n=5$ ). Liver specimens were obtained at necropsy at $1 \mathrm{y}$ of age.

RESULTS: Hepatic trimethylation of lysine 4 of histone $\mathrm{H} 3$ is decreased (CTR/HF 0.87-fold, $P=0.038$; HF/CTR 0.84-fold, $P=$ 0.038), whereas hepatic methyltransferase activity increased by virtue of diet exposure (HF/HF 1.3-fold, $P=0.019)$. Using chromatin immunoprecipitation to determine Npas2 promoter occupancy, we found alterations of both repressive and permissive histone modifications specifically with postweaning HF diet exposure.

CONCLUSION: We found that altered Npas2 expression corresponds with a change in the histone code within the Npas2 promoter.

$\mathbf{S}^{\mathrm{t}}$ tudies on the molecular underpinnings of the developmental origins of health and disease investigate how perturbations in the in utero environment can predispose an individual to disease in adulthood (1). Individuals exposed in the womb to various maternal constraints such as a low-protein diet, maternal caloric restriction, or maternal high-fat (HF) diet were found to have a higher susceptibility to the adult onset of metabolic syndrome than unexposed offspring (2). In light of the current obesity epidemic, study of the long-lasting effects of in utero exposure to a maternal HF diet and maternal obesity is extremely important. How this memory of in utero exposure is maintained remains an unanswered question in the field, and epigenetic modifications in the offspring have logically emerged as potential molecular mediators.

DNA methylation and the removal and addition of posttranslational modifications to the histone proteins are mechanisms the cell utilizes to regulate gene transcription. Although the "histone code" has not been specifically defined, in general, certain modifications are associated with gene expression (permissive modifications), whereas others are associated with gene repression (repressive modifications) (Figure 1a). Acetylated histones, such as $\mathrm{H} 3 \mathrm{~K} 14 \mathrm{ac}$, are traditionally associated with gene expression (3). Trimethylation of lysine 4 of histone H3 $(\mathrm{H} 3 \mathrm{~K} 4 \mathrm{me} 3)$ is also found in the promoter region of active genes (4). However, histone lysine trimethylation does not necessarily correspond with gene activation. Both H3K9me3 and $\mathrm{H} 3 \mathrm{~K} 27 \mathrm{me} 3$ are enriched in promoters of inactive genes (5) (Figure 1a). Histone posttranslational modifications are not permanent. Enzymes such as histone acetyltransferases and histone deacetylases (HDACs) add and remove acetyl moieties from histone lysine residues (6) (Figure 1b). Histone methyltransferases (HMTases) add mono-, di-, or trimethyl groups to histones (7) (Figure 1b). These modifications work in coordination with transcriptional activators and repressors to ensure proper regulation of gene expression.

In recent years, several investigators have shown that epigenetic changes are modifiable with maternal diet constraints (2). Changes in DNA methylation and histone modifications have been reported with exposure to maternal calorie and protein restriction using rat model systems $(8,9)$. In humans, in utero exposure to famine results in changes in DNA methylation, which persist into adulthood (10). Using a mouse model, it has been shown that exposure to maternal methyl supplements results in changes in DNA methylation at specific loci (11). Of note, in each of these studies, the in utero constraint was associated with an increased susceptibility to hypertension and obesity in the offspring in adulthood.

We have previously reported epigenetic, metabolomic, circadian, and pathological changes associated with maternal HF diet exposure in a nonhuman primate model of excess nutrition (12-18). Specifically, we have previously demonstrated that $\mathrm{H} 3 \mathrm{~K} 14 \mathrm{ac}$ is increased in the fetal liver with HF diet exposure, whereas HDAC activity is decreased (12). Further characterization of the fetal liver revealed alterations in the expression of Npas2, an important regulator of circadian genes in peripheral organs such as the liver. This altered expression was accompanied by alterations in the histone code in the promoter of the Npas 2 gene (15). The Npas 2

'Department of Obstetrics and Gynecology, Baylor College of Medicine, Houston, Texas; ${ }^{2}$ Division of Neurosciences, Oregon Health Sciences University, Oregon National Primate Research Center, Beaverton, Oregon; ${ }^{3}$ Division of Reproductive and Developmental Sciences, Oregon National Primate Research Center, Beaverton, Oregon. Correspondence: Kjersti M. Aagaard (aagaardt@bcm.edu) 
a

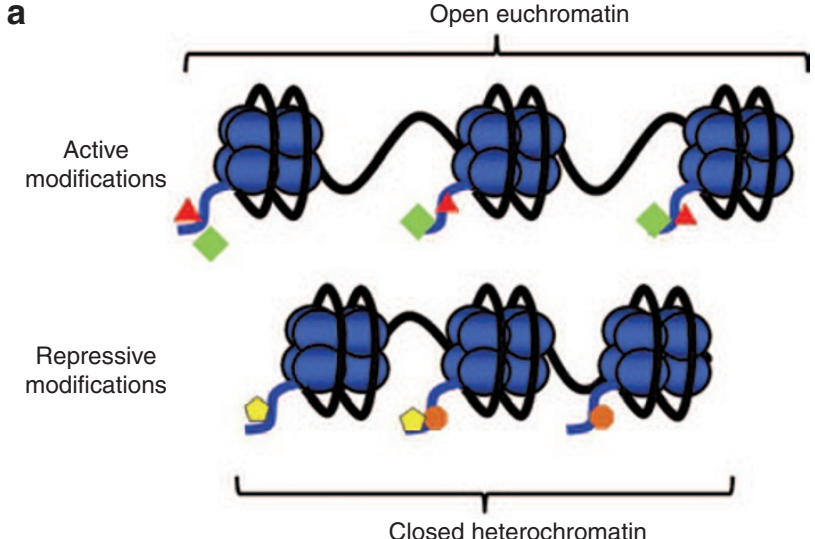

b
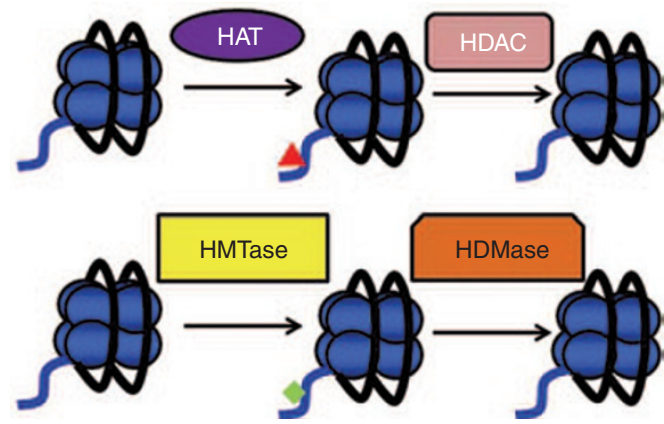

Figure 1. Histone modifications are associated with different chromatin states. (a) Both histone modifications, H3K14ac and H3K4me3, are associated with active, open euchromatin. Both H3K9me3 and H3K27me3 are associated with repressed, closed heterochromatin. (b) Addition of an acetyl group to the $\mathrm{N}$-terminal histone domain is achieved through the enzymatic activity of HATs. These modifications can be removed by HDACs. Similarly, the enzymatic activity of HMTases can add mono-, di-, or trimethyl groups to the histone proteins, and these modifications can be removed by HDMases. HATs, histone acetyltransferases; HDACs, histone deacetylases; HDMases, histone demethylases; HMTases, histone methyltransferases.

promoter contains a transcriptional DNA-binding element and two tandem ROR $\alpha$ response elements (RORE) essential for gene expression (19). In the late-gestation fetus, Npas2 expression is increased with HF diet exposure, and in both temporal and spatial association, H3K14ac is enriched within the RORE.

Study in our macaque model system has revealed effects of the in utero diet exposure that are manifested postnatally. Specifically, offspring show alterations in the serotonergic system and increased anxiety (20). Because we and others have found that epigenetic modifications are modifiable through environmental interactions $(21,22)$, we set out to investigate whether the changes we found in fetal liver would persist postnatally in 1-year-old Japanese macaques. Offspring were exposed to maternal control (CTR) or HF diet in utero and postnatally until weaning at $\sim 7$ mo of age. At weaning, offspring were either assigned to a CTR or a HF diet, yielding four different exposure models (Figure 2, in utero diet/postweaning diet: CTR/CTR; CTR/HF; HF/CTR; and HF/HF). We hypothesized that animals exposed to a HF diet in utero would have an altered histone code, potentially showing an increase in levels

\begin{tabular}{|c|c|c|}
\multicolumn{1}{c}{$\begin{array}{c}\text { In utero } \\
\text { exposure }\end{array}$} & $\begin{array}{c}\text { Postweaning } \\
\text { diet }\end{array}$ & \multicolumn{1}{c|}{ In utero/postweaning } \\
\hline \multirow{2}{*}{ Control } & Control & CTR/CTR \\
\cline { 2 - 3 } & High fat & CTR/HF \\
\hline \multirow{2}{*}{ High fat } & Control & HF/CTR \\
\cline { 2 - 3 } & High fat & HF/HF \\
\hline
\end{tabular}

Figure 2. Model of in utero and postweaning diet exposure in Japanese macaques. Animals were exposed to either a control or a high-fat diet in utero. Animals were delivered at term and were placed on one of the two postweaning diets, either control or high fat. This model yielded four cohorts of diet exposure, depending on the combination of in utero and postweaning diet exposures, designated as in utero/postweaning. $\mathrm{CTR}$, control; HF, high fat.

of H3K14ac similar to fetal life. We also sought to determine whether activity of the histone-modifying enzymes differed by virtue of diet exposure. Finally, we sought to interrogate the Npas 2 promoter in the juvenile animals. We have previously shown that Npas2 expression is increased with postweaning HF diet exposure (15). We sought to determine whether the increase in expression was associated with altered promoter occupancy in our juvenile animals.

\section{RESULTS}

\section{H3K4me3 Is Altered by Virtue of Diet Exposure}

We have previously reported that the fetal histone code at $\mathrm{H} 3 \mathrm{~K} 14 \mathrm{ac}$ is upregulated in association with exposure to maternal HF diet in the fetal liver at gestational day 130 (term $167 \mathrm{~d}$ in the Japanese macaque) (12). This change in histone H3K14ac is associated with hepatic markers of nonalcoholic fatty liver disease in the fetus (13). We have also shown that the increased fetal hepatic triglycerides persisted to postnatal d 180 (13). We sought to determine whether the upregulation in altered histone acetylation also persists postnatally. Using western blotting on acid-extracted histones to detect alterations in the juvenile hepatic histone code, we interrogated the abundance of two permissive (H3K14ac and H3K4me3) and two repressive (H3K9me3 and $\mathrm{H} 3 \mathrm{~K} 27 \mathrm{me} 3)$ histone modifications in our four cohorts of offspring. We found that levels of H3K4me3 changed significantly with differing diet exposures both in utero and postweaning (Figure 3a). Comparing each group with the CTR/CTR (i.e., maternal CTR diet and CTR diet postweaning cohort), we found a significant decrease in H3K4me3 in the CTR/HF group (0.87-fold, $P=0.038$ ). Furthermore, there was a decrease $(0.84$-fold, $P=0.038)$ in H3K4me3 in the HF/CTR. However, in the group chronically exposed to HF diet (HF/HF), there was no significant difference in H3K4me3 levels. Although we have found that fetal H3K14ac levels are altered in utero with HF diet exposure, we did not find a significant change in any of our postweaning exposure cohorts at $1 \mathrm{y}$ of age (Figure $3 \mathbf{b}$ ). Neither H3K9me3 (Figure 3c) nor H3K27me3 (Figure 3d) was significantly altered in any of the cohorts. 
a

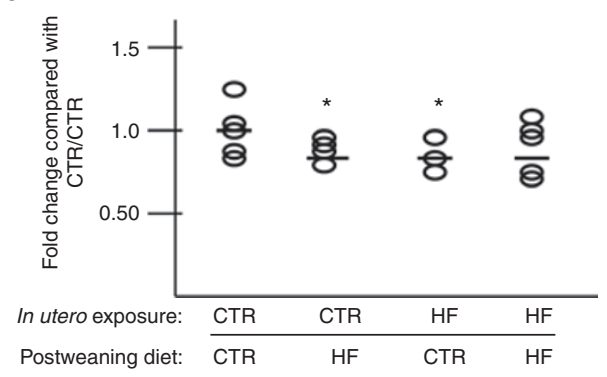

C

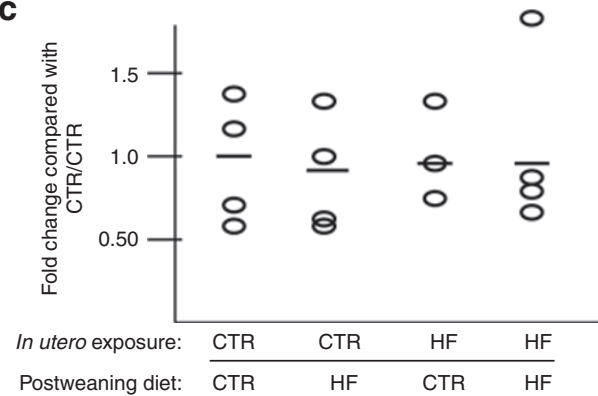

b

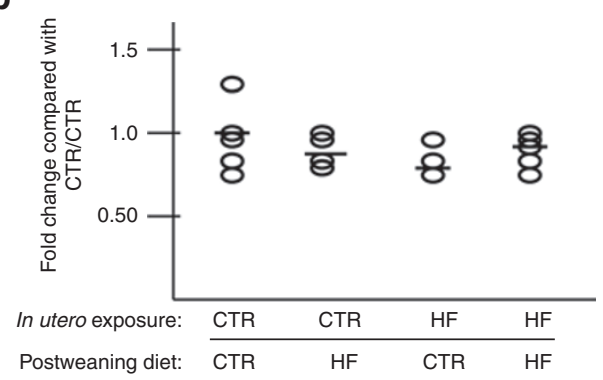

d

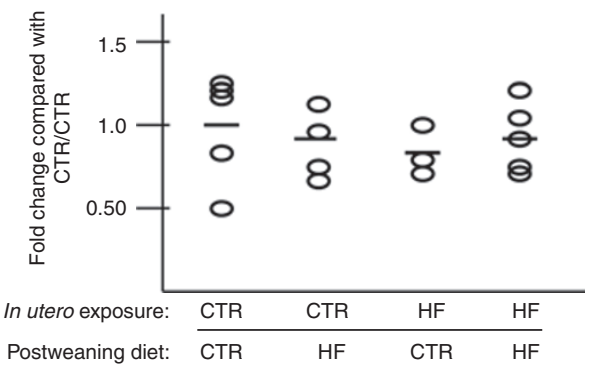

Figure 3. H3K4me3 methylation in the juvenile liver is altered by virtue of diet exposure. Using western blotting of extracted hepatic histones, we found that (a) H3K4me3 is decreased in two exposure models (CTR/HF $(P=0.038)$ and HF/CTR $(P=0.038))$. Levels of $(\mathbf{b})$ H3K14ac, (c) H3K9me3, and (d) H3K27me3 did not change in any of the groups tested. CTR, control; HF, high fat.

Histone H3 Lysine 4 Methyltransferase Activity Is Altered With Chronic HF Diet Exposure

After determining that hepatic H3K4me3 levels were altered in our model system, we sought to determine whether there is a change in H3K4 HMTase activity in hepatic lysates from each of the four cohorts. Using a commercially available kit, which specifically measures only $\mathrm{H} 3 \mathrm{~K} 4$ methyltransferase activity, we found that, indeed, chronic exposure to a maternal and postweaning $\mathrm{HF}$ diet $(\mathrm{HF} / \mathrm{HF})$ is associated with increased HMTase activity (1.34-fold, $P=0.019$ ) (Figure 4a). There were no significant changes in the other cohorts. Because we have previously reported and consistently observed that fetal HDAC activity is altered in utero with maternal HF diet exposure (12), we sought to determine whether this alteration persists postnatally. Consistent with our observations that the juvenile histone code is altered by a maternal HF diet on $\mathrm{H} 3 \mathrm{~K} 4 \mathrm{me} 3$, but not on $\mathrm{H} 3 \mathrm{~K} 14 \mathrm{ac}$, we did not observe significant changes in HDAC activity in our model system (Figure $4 \mathbf{b}$ ).

\section{Promoter Occupancy of the RORE Transcriptional Element of} Npas2 Is Altered With Diet Exposure

We have previously published that $\mathrm{H} 3 \mathrm{~K} 14 \mathrm{ac}$ is enriched in the RORE of Npas 2 in fetal life with HF diet exposure (15). The increase in acetylation, a modification associated with gene expression, was concomitant with an increase in Npas 2 gene expression and demonstrated significant correlation among alterations in promoter occupancy and exon transcription. In juvenile liver, exposure to a postweaning HF diet leads to significant increases in Npas2 expression (15). Because juvenile Npas2 expression levels are increased in both the CTR/ $\mathrm{HF}$ and the HF/HF offspring (15), we sought to interrogate the Npas 2 promoter in the liver of juvenile animals to determine whether promoter occupancy is altered in a manner akin to that observed in the fetus.

Using antibodies specific for the permissive $\mathrm{H} 3 \mathrm{~K} 4 \mathrm{me} 3$, we found this modification was enriched in the HF/HF group (7.7 vs. $15.0 \%, P=0.016$ ) as compared with CTR/CTR (Figure 5a). This change corresponds to the increase in HMTase activity that we found in this cohort (Figure 4a). Using an antibody specific for $\mathrm{H} 3 \mathrm{~K} 14 \mathrm{ac}$, we found that there were no significant changes in any juvenile cohort interrogated (Figure 5b). Of note, both repressive modifications showed depletion in the postweaning HF diet groups. Using an antibody to determine enrichment of $\mathrm{H} 3 \mathrm{~K} 9 \mathrm{me} 3$ at the RORE, we found that the modification is depleted in the cohorts on a postweaning HF diet, CTR/HF (2.7 vs. $0.7 \%, P=0.001)$, and $\mathrm{HF} / \mathrm{HF}(2.7$ vs $0.6 \%, P<0.01$ ) (Figure $5 \mathrm{c}$ ). We found similar results with H3K27me3 (Figure 5d). The modification is depleted in CTR/ $\mathrm{HF}$ (4.6 vs. $3.0 \%, P=0.021)$ and $\mathrm{HF} / \mathrm{HF}(4.6$ vs. $1.9 \%, P<0.01$ ) as compared with CTR/CTR.

\section{DISCUSSION}

How a caloric-dense HF maternal diet ultimately affects the phenotypic outcome of the offspring is unclear. However, studies using nonhuman primates demonstrate that without intervention, the consequences are significant. HF exposure during the prenatal period predisposes offspring to be more susceptible to increased fat mass and insulin resistance (23), cardiovascular reactivity (24), liver dysfunction (25), and anxiety (20) later in life. The molecular mechanisms behind the development of these phenotypes in adulthood are poorly defined. However, we believe that our primate model, examining both in utero and postnatal diet exposures, will yield information as to how these phenotypes emerge. Epigenetic changes, which 


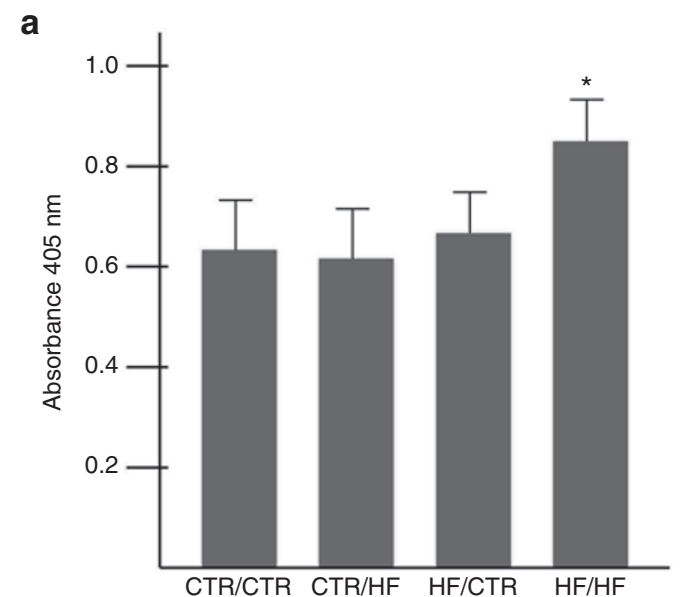

b

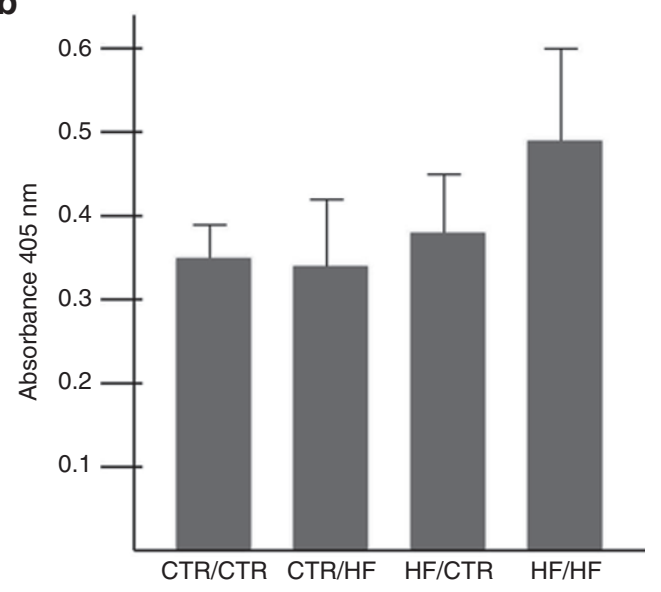

Figure 4. Juvenile hepatic histone methyltransferase (HMTase) activity is increased in HF/HF-exposed animals. Using a commercially available kit, we measured histone H3K4 trimethylase activity. (a) We found that HMTase activity is increased in the HF/HF-exposed group $(P=0.019)$. (b) Histone deacetylase activity in juvenile animals did not change by virtue of diet exposure. ${ }^{*} \mathrm{~A}$ significant $P$ value of $<0.05$. CTR, control; HF, high fat.

occur in utero, may provide a mechanism to maintain a longlasting, stable memory of the in utero experience. Although we have reported epigenetic changes in the fetal liver in our nonhuman primate model system $(12,15,17,18)$, whether these alterations persist and how they parlay into later phenotypic alterations remains to be elucidated.

Our previous epigenetic characterizations of the fetal primate liver exposed to maternal HF diet revealed that H3K14ac is increased as compared with CTR diet-exposed animals (12). We have also determined that this modification is enriched in the promoter of the circadian transcription factor, Npas2, specifically in the fetal livers of HF diet-exposed animals (15). This change in promoter occupancy was also accompanied by disrupted expression of Npas2 in the HF diet-exposed fetal liver (15). Analysis of gene expression in the juvenile liver showed that Npas 2 regulation is disrupted only in the animals that consumed a HF diet postweaning, regardless of their in utero exposure.

Although it has been shown that HF diet feeding disrupts circadian rhythm and gene expression in the mouse (26), we were the first to show that in utero exposure to HF diet disrupts the peripheral circadian rhythm in the fetus. Many elegant studies in animal models have demonstrated a cyclicity of histone modifications in the promoter regions of these circadian-regulated genes (27). Over a 24 -h period, such promoters see a change in histone acetylation caused by the interaction of NPAS2 with the histone acetyltransferase p300 (28). Abundance of $\mathrm{H} 3 \mathrm{~K} 4$ methylation in specific promoters also changes over a 24 -h period because of interactions of the circadian genes with the HMTase mixed lineage leukemia (29). In the current study, we wanted to determine whether the HF diet-dependent changes in both histone modification abundance and localization to the Npas 2 promoter that we observed in fetal life were maintained postnatally in our juvenile cohort.

We hypothesized that animals exposed in utero to a HF diet may maintain the increased H3K14 acetylation postnatally. Using histone modification-specific antibodies, we measured H3K14ac abundance in juvenile liver. Consistent with our prior observations in the fetal liver, we observed that the histone code in juvenile animals is modified by both a maternal and a postweaning HF diet. Of interest, however, were the site and nature of these histone modifications. Specifically, we found that in the juveniles, H3K14 acetylation did not differ significantly with maternal or postnatal HF diet exposure as compared with CTR/CTR (Figure $3 b$ ). However, another permissive modification, H3K4me3, was altered by virtue of diet exposure. Specifically, we found that levels were slightly but significantly decreased in both the CTR/HF and the HF/CTR groups as compared with CTR/CTR (Figure 3a). Because H3K4me3 is altered with both CTR and HF in utero exposures, we cannot conclude that the in utero environment is responsible for the reprogramming of the juvenile hepatic histone code. In addition, chronic exposure to a HF diet cannot be implicated because the HF/HF group did not show a difference in abundance of this modification. Potentially, a disconnect between in utero and postnatal environments may contribute to the alterations in $\mathrm{H} 3 \mathrm{~K} 4 \mathrm{me}$. Interrogation of the abundance of two repressive histone modifications, H3K9me3 and H3K27me3, revealed no difference (Figure 3c,d). Taken together and in consideration of our prior observations, these findings suggest that the fetal histone code is modified at H3K14 in association with maternal HF diet intake, whereas the juvenile code is modified at $\mathrm{H} 3 \mathrm{~K} 4 \mathrm{me} 3$. We find it of significance that H3K14 appears to be modifiable specifically in fetal life; this finding is consistent with others' observations regarding developmental specificity to the histone code (30).

Our unique model system gives us insights into the potential mechanisms that may lie behind the discrepancy we observe between our fetal and juvenile cohorts. We have recently published that the abundance and activity of the HDAC, SIRT1, is decreased in the fetal liver with HF diet exposure (17). We also found that SIRT1 deacetylates H3K14ac in vitro. Although we believe that SIRT1 may play a role in the increased H3K14ac seen in fetal life, we have not tested the abundance and activity of SIRT1 in the juvenile liver. It would be of interest to test whether these changes in SIRT1 level and activity are stable 
a

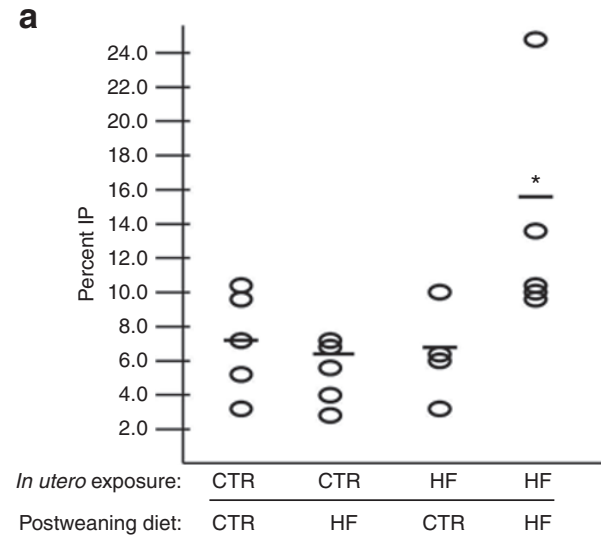

C

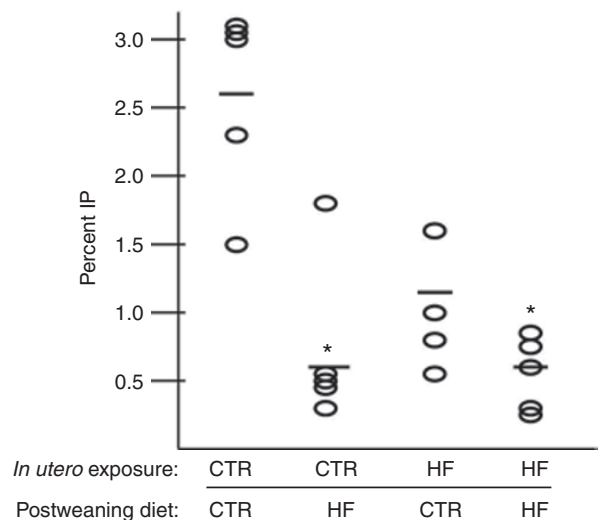



d



Figure 5. The RORa response element (RORE) of Npas2 is depleted for repressive modifications with postweaning exposure to a HF diet. Using chromatin immunoprecipitation for various histone modifications, we interrogated the occupancy of the RORE of Npas2 in juvenile liver. (a) We found that H3K4me3 is enriched in the RORE of the HF/HF-exposed animals $(P=0.016)$. (b) H3K14ac levels are not altered with any diet condition. (c) H3K9me3 is depleted in CTR/HF $(P=0.001)$ and HF/HF $(P<0.01)$. (d) H3K27me3 is similarly depleted in CTR/HF $(P=0.021)$ and HF/HF $(P<0.01) .{ }^{*} \mathrm{~A}$ significant $P$ value of $<0.05$.

CTR, control; $\mathrm{HF}$, high fat; IP, immunoprecipitate.

in the juvenile liver and, if not, which specific HMTases are responsible for the increase in $\mathrm{H} 3 \mathrm{~K} 4 \mathrm{me} 3$ observed in the juvenile liver.

Differences between the fetal and postnatal characteristics may also play a role in changes in the epigenetic milieu of the liver during development. In our nonhuman primate (NHP) model system, we have found that fetuses exposed to a maternal HF diet weigh $10 \%$ less at the beginning of the third trimester than those exposed to a CTR diet (13). This is probably caused by lower lean body mass. However, at both postnatal d 30 and 180, these animals weigh the same as CTR dietexposed animals but have almost twice the body fat, as determined using dual-energy X-ray absorptiometry, as compared with CTRs. In these juvenile animals, we have also found an increased plasma insulin level and an abnormal cardiovascular morphology (31). Perhaps the accumulation of white adipose tissue and the disparity in body composition from the third trimester to juvenile life in these animals is helping to orchestrate the alterations we observe in the hepatic histone code.

We sought to determine the mechanism behind this decrease in $\mathrm{H} 3 \mathrm{~K} 4 \mathrm{me} 3$ levels and hypothesized that this decrease could be the result of lower levels of HMTase activity (32). Using a commercially available kit, we specifically measured histone $\mathrm{H} 3$ lysine 4 trimethyltransferase activity from hepatic lysates. Rather than finding decreased HMTase activity in the CTR/HF and HF/CTR groups, which would account for the decreased trimethylation, we found that activity was significantly increased in the HF/HF group (Figure 4a). This could potentially explain why this cohort did not exhibit a decrease in H3K4me3 abundance by western blot as the increased activity may be, resultantly, affecting the measurement of histone methyl group stability. We have also previously shown that HDAC activity is decreased in HF diet-exposed fetal liver as compared with CTR (12). We sought to determine whether this decrease in activity persists postnatally with in utero HF diet exposure. We found that HDAC activity did not change in any of the groups tested as compared with CTR/CTR, which is consistent with our observations regarding acetylation (Figure 4b).

On the basis of our previous findings that the permissive modification, H3K14ac, was enriched in the promoter of Npas 2 in the HF diet-exposed fetal liver, we had hypothesized that such a permissive modification may be similarly enriched in the juvenile cohort. According to our findings in this study that the abundance of $\mathrm{H} 3 \mathrm{~K} 4 \mathrm{me} 3$ is altered whereas that of $\mathrm{H} 3 \mathrm{~K} 14 \mathrm{ac}$ is not, we hypothesized that perhaps H3K4me3 
would be enriched in the Npas 2 promoter. Because we have found that expression of Npas 2 in juvenile animals is increased with a postweaning HF diet, and not by in utero HF diet exposure (15), we believed that H3K4me3 would be enriched specifically in these two groups. However, we found increased enrichment only in the HF/HF group (Figure 5a). Our chromatin immunoprecipitation analysis utilizing an antibody for H3K14ac did not reveal the differential promoter occupancy we observed in fetal life. However, using antibodies against two repressive modifications, differential enrichment in the RORE of Npas 2 is demonstrated. Both H3K9me3 and H3K27me3 are depleted in the CTR/HF and HF/HF groups, which also show increased expression of Npas2 (Figure $5 \mathbf{c}, \mathbf{d}$ ). Although these results were unexpected, the depletion of repressive modifications in these two groups correlates with the higher expression of Npas2.

We have previously characterized epigenetic changes to the fetal hepatic histone code. We found that H3K14ac is an important molecular mediator in the fetal liver exposed to maternal HF diet. However, given our current data, we speculate that this modification may be specific for fetal life. In our four postweaning diet exposure groups, we found that only H3K4me3 abundance is altered by virtue of diet exposure in juvenile liver. Furthermore, the change in HDAC activity in fetal life did not persist, but instead we found an increase in $\mathrm{H} 3 \mathrm{~K} 4$ trimethylase activity. Finally, we found that rather than the Npas2 RORE being enriched for permissive histone modifications in the cohorts that have increased Npas 2 expression, it is depleted for repressive modifications.

Our data reveal that postweaning diet influences the epigenomic signature of the individual. The epigenetic changes we have reported in fetal life, namely, increased H3K14ac abundance and decreased HDAC activity with HF diet exposure, do not persist postnatally. By contrast, we report that novel histone modifications are altered by virtue of postweaning diet exposure. We found alterations in the abundance of H3K4me3 and in HMTase activity and observed differential promoter occupancy of repressive histone modifications. Because we cannot conclude that site-specific epigenetic alterations, which occur during fetal life, maintain the memory of the in utero exposure, we must determine how epigenetic changes in fetal life influence the alterations postnatally.

Although we have not studied the circadian behavior of our juvenile cohort, we have found that the histone occupancy of the promoter region, as well as the expression level of Npas2, is altered with HF diet exposure in fetal and juvenile life. We believe that these alterations to an important circadian rhythm transcription factor warrant further investigation into the transcriptional, epigenetic, and perhaps even behavioral consequences of HF diet consumption during pregnancy. The findings herein lay the foundation for future investigations and suggest that although the histone code is developmental stage specific, our initial overarching observations persist. In summary, a maternal HF diet modifies both the fetal and the postnatal histone code, and postweaning exposure to a HF diet similarly modifies histones in a type- and site-specific manner.

\section{METHODS}

\section{Study Design}

All animal procedures were approved by the Oregon National Primate Research Center Institutional Animal Care and Use Committee and conformed to National Institutes of Health guidelines on the ethical use of animals. Briefly, weight- and age-matched adult female Japanese macaques (Macaca fuscata) were fed a CTR or HF for up to $4 \mathrm{y}$. The CTR diet (Fiber Balanced Monkey Diet 5000; Purina Lab Diet, St. Louis, MO) provided $13 \%$ of calories from fat and the HF diet (Test Diet, 5LOP; Purina Lab Diet) provided 32\% of calories from fat and was supplemented with calorically dense treats. The HF diet represents a typical Western diet in regard to saturated fat content. Monkeys were housed in indoor/outdoor pens in groups of $\sim 12$ individuals (male:female ratio 2:10). Monkeys had ad libitum access to food and water.

To generate juvenile offspring ( 1 y of life), a cohort of dams $(n=$ 18) progressed to term (e167). Dams had no more than two prior pregnancies before delivering the subjects of this study. To evaluate the effects of the postweaning diet, juveniles were grouped by diet exposure in utero and by postweaning diet to yield four offspring exposure models (Figure 2, in utero diet/postweaning diet: i.e., CTR/ CTR, $n=5$; CTR/HF, $n=4$; HF/CTR, $n=4$; HF/HF, $n=5$ ). Juvenile liver samples were obtained at 5 mo postweaning when animals were killed at $\sim 1$ y of age. Briefly, animals were fasted for $\sim 4 \mathrm{~h}$ before necropsy and sedated with $15-25 \mathrm{mg} / \mathrm{kg}$ of ketamine intramuscularly. The animals were deeply anesthetized with a surgical dose of sodium pentobarbital $(25-30 \mathrm{mg} / \mathrm{kg}$ intravenous) by veterinary staff and then exsanguinated. Liver specimens were then snap frozen in liquid nitrogen and stored at $-80^{\circ} \mathrm{C}$ until use. All procedures involving animals underwent an extensive Institutional Animal Care and Use Committee review process at Oregon Health \& Science University and Baylor College of Medicine.

\section{Western Blotting}

Histones were extracted as previously described (12). Samples were run on $18 \%$ Tris-glycine gels (Invitrogen, Carlsbad, CA), transferred to PVDF (BioRad, Hercules, CA), and incubated overnight in primary antibody (Millipore, Billerica, MA) at a dilution of 1:1,000. The blot was incubated for $45 \mathrm{~min}$ in secondary antibody (antirabbit IgG; Cell Signaling, Beverly, MA) and visualized using chemiluminescence (Perkin-Elmer, Waltham, MA). Blots were stripped with stripping buffer (Pierce, Rockford, IL) and reprobed with GAPDH antibody (Abcam, Cambridge, MA) to normalize loading. Of note, for the western blot experiments, the HF/CTR group had an $n$ of 3 .

\section{HDAC and HMTase Assays}

Nuclear extracts were prepared and HDAC assays (Biomol, Farmingdale, NY) were performed as previously described (12). For HMTase assays, $80 \mu \mathrm{g}$ of hepatic lysate was used and assay was performed according to manufacturer conditions (Epigentek, Farmingdale, NY).

\section{Chromatin Immunoprecipitation}

Chromatin immunoprecipitation was performed as previously described (15). Briefly, $100 \mu \mathrm{g}$ of chromatin was incubated overnight with histone modification-specific antibodies (Millipore). Ten percent of the total chromatin used was set aside as the "input" CTR. Bound chromatin was precipitated using Protein A Dynal beads (Invitrogen), eluted with 10\% sodium dodecyl sulfate, and purified with QIAquick columns (Qiagen, Valencia, CA). PCR reactions were set up using primers as described (15).

\section{Analysis}

A Student's $t$ test was used for statistical significance for all assays. To determine percentage IP, samples were first normalized to the input for each sample. Percentage immunoprecipitate (IP) was calculated as $100^{\star} 2^{\wedge}($ Input-IP).

\section{ACKNOWLEDGMENT}

Data from this paper were presented at the 31st Annual Meeting of the Society for Maternal Fetal Medicine, San Francisco, California, 10 February 2011. 


\section{STATEMENT OF FINANCIAL SUPPORT}

This work was supported by the National Institutes of Health (NIH, Bethesda, MD) Director New Innovator Award (DP21200D001500-01 to K.M.A.) National Institute of Child Health and Human Development/National Institute of Diabetes and Digestive and Kidney Diseases (NICHD/NIDDK) Grant R01DK080558-01 (to K.M.A.), and the NIH Research Education and Career Horizon Institutional Research and Academic Career Development Award (REACH IRACDA) K12 GM084897 (to M.A.S.).

Disclosure: The authors have neither conflicts of interest nor financial disclosures.

\section{REFERENCES}

1. Gluckman PD, Hanson MA, Cooper C, Thornburg KL. Effect of in utero and early-life conditions on adult health and disease. $\mathrm{N}$ Engl $\mathrm{J}$ Med 2008;359:61-73.

2. Suter MA, Aagaard-Tillery KM. Environmental influences on epigenetic profiles. Semin Reprod Med 2009;27:380-90.

3. Nakayama J, Rice JC, Strahl BD, Allis CD, Grewal SI. Role of histone H3 lysine 9 methylation in epigenetic control of heterochromatin assembly. Science 2001;292:110-3.

4. Ruthenburg AJ, Allis CD, Wysocka J. Methylation of lysine 4 on histone H3: intricacy of writing and reading a single epigenetic mark. Mol Cell 2007;25:15-30.

5. Wang Z, Schones DE, Zhao K. Characterization of human epigenomes. Curr Opin Genet Dev 2009;19:127-34.

6. Peserico A, Simone C. Physical and functional HAT/HDAC interplay regulates protein acetylation balance. J Biomed Biotechnol 2011;2011:371832.

7. Kota SK, Feil R. Epigenetic transitions in germ cell development and meiosis. Dev Cell 2010;19:675-86.

8. Lillycrop KA, Phillips ES, Jackson AA, Hanson MA, Burdge GC. Dietary protein restriction of pregnant rats induces and folic acid supplementation prevents epigenetic modification of hepatic gene expression in the offspring. J Nutr 2005;135:1382-6.

9. Lillycrop KA, Slater-Jefferies JL, Hanson MA, Godfrey KM, Jackson AA, Burdge GC. Induction of altered epigenetic regulation of the hepatic glucocorticoid receptor in the offspring of rats fed a protein-restricted diet during pregnancy suggests that reduced DNA methyltransferase- 1 expression is involved in impaired DNA methylation and changes in histone modifications. Br J Nutr 2007;97:1064-73.

10. Tobi EW, Lumey LH, Talens RP, et al. DNA methylation differences after exposure to prenatal famine are common and timing- and sex-specific. Hum Mol Genet 2009;18:4046-53.

11. Cooney CA, Dave AA, Wolff GL. Maternal methyl supplements in mice affect epigenetic variation and DNA methylation of offspring. J Nutr 2002;132:Suppl 8:2393S-400S.

12. Aagaard-Tillery KM, Grove K, Bishop J, et al. Developmental origins of disease and determinants of chromatin structure: maternal diet modifies the primate fetal epigenome. J Mol Endocrinol 2008;41:91-102.

13. McCurdy CE, Bishop JM, Williams SM, et al. Maternal high-fat diet triggers lipotoxicity in the fetal livers of nonhuman primates. J Clin Invest 2009;119:323-35.

14. Cox J, Williams S, Grove K, Lane RH, Aagaard-Tillery KM. A maternal high-fat diet is accompanied by alterations in the fetal primate metabolome. Am J Obstet Gynecol 2009;201:281.e1-9.
15. Suter M, Bocock P, Showalter L, et al. Epigenomics: maternal high-fat diet exposure in utero disrupts peripheral circadian gene expression in nonhuman primates. FASEB J 2011;25:714-26.

16. Frias AE, Morgan TK, Evans AE, et al. Maternal high-fat diet disturbs uteroplacental hemodynamics and increases the frequency of stillbirth in a nonhuman primate model of excess nutrition. Endocrinology 2011;152:2456-64.

17. Suter MA, Chen A, Burdine MS, et al. A maternal high-fat diet modulates fetal SIRT1 histone and protein deacetylase activity in nonhuman primates. FASEB J 2012;26:5106-14.

18. Suter MA, Sangi-Haghpeykar H, Showalter L, et al. Maternal high-fat diet modulates the fetal thyroid axis and thyroid gene expression in a nonhuman primate model. Mol Endocrinol 2012;26:2071-80.

19. Yamamoto T, Nakahata Y, Soma H, Akashi M, Mamine T, Takumi T. Transcriptional oscillation of canonical clock genes in mouse peripheral tissues. BMC Mol Biol 2004;5:18.

20. Sullivan EL, Grayson B, Takahashi D, et al. Chronic consumption of a highfat diet during pregnancy causes perturbations in the serotonergic system and increased anxiety-like behavior in nonhuman primate offspring. J Neurosci 2010;30:3826-30.

21. Waterland RA, Jirtle RL. Early nutrition, epigenetic changes at transposons and imprinted genes, and enhanced susceptibility to adult chronic diseases. Nutrition 2004;20:63-8.

22. Lillycrop KA, Burdge GC. Epigenetic changes in early life and future risk of obesity. Int J Obes (Lond) 2011;35:72-83.

23. Shankar K, Harrell A, Liu X, Gilchrist JM, Ronis MJ, Badger TM. Maternal obesity at conception programs obesity in the offspring. Am J Physiol Regul Integr Comp Physiol 2008;294:R528-38.

24. Rudyk O, Makra P, Jansen E, Shattock MJ, Poston L, Taylor PD. Increased cardiovascular reactivity to acute stress and salt-loading in adult male offspring of fat fed non-obese rats. PLoS ONE 2011;6:e25250.

25. Zhang X, Strakovsky R, Zhou D, Zhang Y, Pan YX. A maternal high-fat diet represses the expression of antioxidant defense genes and induces the cellular senescence pathway in the liver of male offspring rats. J Nutr 2011;141:1254-9.

26. Kohsaka A, Laposky AD, Ramsey KM, et al. High-fat diet disrupts behavioral and molecular circadian rhythms in mice. Cell Metab 2007;6:414-21.

27. Feng D, Lazar MA. Clocks, metabolism, and the epigenome. Mol Cell 2012;47:158-67.

28. Etchegaray JP, Lee C, Wade PA, Reppert SM. Rhythmic histone acetylation underlies transcription in the mammalian circadian clock. Nature 2003;421:177-82.

29. Katada S, Sassone-Corsi P. The histone methyltransferase MLL1 permits the oscillation of circadian gene expression. Nat Struct Mol Biol 2010;17:1414-21.

30. Pinney SE, Simmons RA. Epigenetic mechanisms in the development of type 2 diabetes. Trends Endocrinol Metab 2010;21:223-9.

31. Fan L, Lindsley SR, Comstock SM, et al. Maternal high-fat diet impacts endothelial function in nonhuman primate offspring. Int J Obes (Lond) 2013;37:254-62.

32. Teperino R, Schoonjans K, Auwerx J. Histone methyl transferases and demethylases; can they link metabolism and transcription? Cell Metab 2010;12:321-7. 